\title{
El impacto de una estrategia propuesta basada en la orientación de exploración en el desarrollo de habilidades de habla árabe entre los primeros estudiantes intermedios
}

\author{
O impacto de uma estrategia baseada na orientacao da exploracao no \\ desenvolvimento de habilidades de ensino entre os principaes estudiantes intermedios
}

Aceptado: 09 de mayo de 2019

\section{Resumen}

La investigación actual apunta a desarrollar las habilidades de hablar el idioma árabe en los estudiantes de primer grado. La muestra de estudio consistió en estudiantes de escuela intermedia en el centro de la ciudad de Babil para el año académico 2017-2018, la muestra de investigación consistió en (24) estudiantes de los estudiantes de primer grado que representan al grupo experimental y (24) estudiantes que representan el control En grupo, las herramientas de investigación consistían en una lista de habilidades para hablar y una nota para hablar. Después de completar el experimento, el investigador concluyó que la estrategia propuesta basada en el enfoque orientado al Movimiento Scout en el desarrollo de las habilidades de habla árabe de los estudiantes de primer grado se logró a través de la superioridad del grupo experimental sobre los estudiantes del grupo de control y esto se refleja en los resultados de la aplicación posterior de la tarjeta de notas habilidades de habla.

Palabras clave: Idioma árabe. Estudiantes, habilidades de habla.

\section{Resumo}

A investigação real apunta a desenvolver as habilidades de canto a língua árabe nos estudiantes de primer grado. A série de estudo consistiu em estudantes de carreira intermediária no centro da cidade de Babil para o ano académico 2017-2018, a pesquisa de mestrado consistiu em (24) estudiosos dos estudantes de primer grado que representaram o grupo experimental y (24)) estudiantes that representan el control group, herramientas de investigación consisting en una lista de skills para hablar y una nota para

\footnotetext{
${ }^{1}$ Facultad de Educación para las Humanidades, Universidad de Babilonia, Ministerio de Educación Superior e Investigación Científica, Iraq.

${ }^{2}$ Profesor Asistente. Facultad de Educación para las Humanidades, Universidad de Babilonia, Ministerio de Educación Superior e Investigación Científica, Iraq.
} 


\section{ORANGE JOURNAL}

hablar. Depois de completar o experimento, o investigador concluiu a estratégia de propagar a partida no enfoque orientado ao Movimento Escoteiro no desenvolvimento das habilidades de expressão árabe dos pesquisadores de primer grado se logrou o sucesso da superioridade do grupo experimental sobre os estudantes do ensino médio grupo de controle e está em jogo nos resultados da aplicação posterior da tabela de notas de habilidades de habla.

Palavras-chave: Idioma árabe. Estudiantes, habilidades de habla.

\section{Introducción}

\section{Primero: Problema de investigación:}

La debilidad del idioma árabe comenzó a aparecer ahora, puede deberse al resultado de nuestro sistema educativo que se enfoca en el adoctrinamiento y la conservación 1, otra razón importante para este problema es la falta de conciencia de los estudiantes en lectura y escritura y el uso adecuado de (Meri y Al-hailah, 2002: 251). Además, el dialecto coloquial es muy común durante la enseñanza y la falta de oportunidades de inversión para capacitarse en el idioma árabe estándar en las lecciones de las otras lecciones de árabe 1. Varios educadores atribuyen el problema de la expresión a otra razón que radica en la falta de interés en asociar la expresión con ramas del otro idioma árabe, lo que hace que las lecciones de expresión no estimulen la motivación de los estudiantes para hablar o escribir, sin embargo, obtener la capacidad de expresar una hermosa expresión es el resultado final del aprendizaje del idioma árabe (Madkour, 2009: 270). Otros han argumentado que la lectura, que incluye elementos de comprensión, crítica y gusto ... es un elemento clave y un requisito previo para el éxito del maestro en el empleo de habilidades lingüísticas. Por lo tanto, el investigador decidió realizar un estudio destinado a identificar el efecto de una estrategia propuesta basada en el enfoque de exploración en el desarrollo de habilidades de habla árabe entre los primeros estudiantes de clase intermedia.

\section{Segundo: La importancia de la investigación:}

El lenguaje es un gran verso de los versos de Alá, el poderoso, el poderoso, como el Señor de la Gloria se ha referido a él en Su Noble Libro "Holly Qur'an": (Entre sus signos está la creación de los cielos y el tierra, y la diferencia de sus lenguas y colores, hay signos en esto para los mundos ...), el lenguaje es una gracia que Dios distinguió de entre todos los demás seres, a través de la cual el hombre logró hacer y desarrollar su vida, a través del cual se llevó a cabo la comunicación de ideas y culturas, y las civilizaciones y naciones continuaron.

Es la única herramienta que permite a un individuo ingresar al alcance de la sociedad en la que vive. De lo contrario, el individuo permanecerá encerrado en aislamiento social (Hassan, 2001: 17). Dado que el lenguaje es el instrumento de expresión, el árabe es el mejor instrumento para la expresión y divulgación. Es un lenguaje flexible, con la capacidad de derivar y generar las palabras que usted desarrolla y adquiere significados metafóricos y terminológicos que no se conocían antes (Ayachi, 1977: 64). 
El árabe es un idioma antiguo de una nación antigua. Se distingue de los otros idiomas. Es el lenguaje del "Holly Qur'an", que está garantizado por el Señor de la Gloria y la Preservación en su libro de gloria que dice :( Hemos bajado nuestro libro y lo tenemos para los guardianes (Al-Hager / 9), en este sentido, el Dr. Jawaad afirma: (El idioma árabe está viviendo con una gran cantidad de problemas diferentes, solución difícil y tratamiento difícil, y estos problemas radican en: Términos, definiciones, gramática y desembolso ... y estos los problemas no eran para el Sagrado Corán y la gran literatura verbal de los olores del alfabeto árabe, si no hubiera "Sagrado Corán" y la gran literatura verbal del alfabeto árabe, y se basara en los temas, se convertiría en algo histórico los idiomas se enseñan solo cuando es necesario, y el sonido no se habla solo después del costo y el sufrimiento.

Aprender en una forma de descubrimiento es muy importante en el proceso de aprendizaje educativo. Glaser cree que aprender por descubrimiento ayuda a descubrir a los aprendices de apego, concepto o regla (Jaber, 2005a, 213).

Al-Sherbini explicó que el aprendizaje por descubrimiento es capaz de lograr los siguientes asuntos educativos importantes.

1. Desarrollar las habilidades mentales de los alumnos, tales como clasificación, reconocimiento de similitudes y diferencias, diferenciación entre información.

2. Los alumnos deben ir más allá del conocimiento y los datos disponibles.

3. Asistir en la formación de estudiantes con la capacidad de innovar, inventar y producir conocimiento.

Al hablar sobre las habilidades del idioma árabe para escuchar, hablar, leer y escribir, descubrirá que hablar es uno de los colores más importantes de la actividad lingüística de adultos y niños (Madkour, 2008: 111). La importancia de hablar en árabe, debe prestar atención a enseñar a los estudiantes sus habilidades, de modo que enseñar habilidades de habla no sea fácil; requiere planificación y establecimiento de objetivos específicos, especialmente cuando hablar no ocurre repentinamente, sino que está precedido por varios procesos de excitación, pensamiento, formulación y pronunciación. Cuando el individuo se cría, comienza a pensar en lo que va a decir y a reunir ideas y organizarlas mentalmente. Habla sin darse el tiempo suficiente para pensar en lo que dirá. Sus palabras a menudo no tienen sentido y están desorganizadas (Jamalwa Akharoon, 2006: 204)

1. El método de aprendizaje por descubrimiento ayuda a simplificar y aclarar en gran medida muchas lecciones difíciles.

2. Es un método basado en inducción e inferencia.

3. Necesitas ser un maestro y un investigador renovado familiarizado con todo lo que es nuevo.

4. Calificar al maestro lo suficientemente bien como para poder administrar y controlar el aula al aplicar cualquiera de los diferentes métodos de enseñanza, incluido el método de descubrimiento de aprendizaje

5. Aquí viene el enfoque de exploración y los beneficios del alumno en el desarrollo de habilidades lingüísticas: 


\section{ORANGE JOURNAL}

La importancia de la investigación actual se puede resumir de la siguiente manera:

1. La importancia del lenguaje para el hombre como principal instrumento de expresión.

2. La importancia del idioma árabe, porque es el idioma espiritual de la nación islámica y el idioma del patrimonio cultural de la nación árabe.

3. La importancia de las actividades en general después de la de los conceptos educativos modernos, y las actividades del lenguaje y la absorción en particular, lo que ayuda a los estudiantes a usar el idioma de forma natural y desarrollar su riqueza lingüística y les ayuda a comprender el texto, la asimilación y el gusto.

4. Lo que representa la lección de lectura de gran importancia entre las ramas del idioma árabe, y lo que proporciona desde un área amplia para la aplicación de estas actividades de ambos tipos, y el investigador cree que estas actividades estudian la lección e invierten.

5. La importancia de la expresión como el objetivo de estudiar las ramas de la lengua árabe combinada, y el único medio de comunicación entre personas en diferentes países y naciones y preservar el equilibrio cultural y cultural y transferirlo a las generaciones futuras.

\section{Objetivos de investigación}

La investigación reciente tiene como objetivo:

1. El impacto de la estrategia orientada al Movimiento Scout en el desarrollo de habilidades de habla árabe para los estudiantes de primer nivel intermedio.

2. Determinar las habilidades orales apropiadas para los estudiantes en el primer grado intermedio.

3. Desarrollar las habilidades de habla apropiadas para los estudiantes en el primer grado intermedio.

\section{Los límites de la investigación:}

La investigación actual está determinada por una muestra de estudiantes de secundaria en la escuela intermedia Al-Hakim para los estudiantes de primer grado, la ciudad de Babil en el año académico 2017-2018.

\section{Terminología:}

1. Estrategia orientada al Movimiento Scout: el aprendizaje se logra a través de procesos mentales selectivos de alto nivel a través de los cuales la información proporcionada se analiza, se reinstala y se transforma en nuevas imágenes, con el objetivo de alcanzar información y conclusiones previamente desconocidas, así como los medios y métodos utilizados por los humanos utilizando sus recursos mentales y físicos (Jaber, 2005b, 212). 
Habilidades para hablar en dos idiomas: el investigador sabe que el procedimiento es la habilidad de los estudiantes en la escuela intermedia de usar un idioma árabe fluido y de expresarlo lingüísticamente.

\section{Referentes teóricos:}

Concepto orientado al Movimiento Scout: origen y mejora.

La idea de aprender del descubrimiento se remonta a la antigüedad cuando Sócrates lo usó con sus discípulos para empujarlos a descubrir sus valores, conocimiento y virtud, y lo llamó el método socrático en relación con el filósofo Sócrates. Este método continuó en forma de diálogos con su alumno Platón y Aristóteles, 2013) http://www.albayan.ae/science-today/studies-research/2013-03-24-1.1847694.

\section{Concepto orientado al Movimiento Scout: origen y evolución}

Este método ha surgido en la educación islámica, donde el Profeta (la paz y las bendiciones de Allah desciendan sobre él) usó el método de diálogo y razonamiento para llamar la atención del "Sahaabah" y guiar su pensamiento para comprender lo que quiere enseñarles. Las enseñanzas de la religión islámica. Esto es lo que encontramos en los "hadices" del Profeta (que la paz y las bendiciones de Allah sean con él) En otras palabras, hay muchos otros "haadeeths". Ibrahim bin Hamza dijo que me dijera que el hijo de Hazem y Aldrawardi Yazid significa Ibn Abdullah bin Hade de Muhammad ibn Ibrahim de Abu Salamah bin Abdul Rahman, de Abu Hurayrah que escuchó al Mensajero de Allah (la paz sea con él) diciendo que usted ve si un río al lado de una puerta en uno de ustedes, lavándose todos los días Cinco veces que no diga, manténgase alejado del estiércol, dijeron que no le ocultaran nada, dijo, es como las cinco oraciones, el Dios borra los pecados por ellos "(Libro de tiempos de oración - la puerta del número de expiación de las cinco oraciones Hadith 505). Los eruditos musulmanes siguieron el enfoque del Profeta (que la paz y las bendiciones de Allah sean con él), incluido Ibn Khaldun, quien pidió no confiando en memorizar en la educación y en dirigir al individuo a adquirir conocimiento de sus fuentes para poder usarlo de por vida (Al-Amoudi, 2003).

"En los tiempos modernos, la educación ha surgido a través del descubrimiento en una serie de direcciones educativas como un llamado general para el uso del descubrimiento centrado en el estudiante. Jean-Jacques Rousseau, que pidió un descubrimiento absoluto y gratuito con el niño, inició los movimientos educativos. dejando al niño a la naturaleza para obtener conocimiento sin depender de los demás. En 1960, él publicó varios libros que tratan sobre sus puntos de vista educativos sobre la naturaleza de su enseñanza y su filosofía educativa. La mayoría de sus ideas fueron apoyadas por educadores (Saeghi, 50, 2006). Brunner fue muy influenciado por Jean Piaget (1963). Se dio cuenta de que los niños son activos en la exploración e interpretación del mundo y se centró en la importancia de la acción y la resolución de problemas. Él cree que los niños exploran el entorno tratando con objetos concretos, experimentando con ideas y devolviendo su comprensión de conceptos o teorías de maneras que no son las mismas que usan los científicos. (Traducción de Mohammad Al-Jawad, 44), mientras que el lanzamiento de la palabra aprendizaje por descubrimiento vuelve a Jerome Brunner (Muhammad, 2009, 47). 


\section{ORANGE JOURNAL}

La importancia del aprendizaje por descubrimiento:

Aprender en una forma de descubrimiento es muy importante en el proceso de aprendizaje educativo. Glisser cree que aprender por descubrimiento ayuda a descubrir la conexión, el concepto o la regla del estudiante (Jaber, 2005b, p. 213).

1. Al-Sherbini (2009) explicó que el aprendizaje por descubrimiento es capaz de lograr los siguientes asuntos educativos importantes:

2. Desarrollar las habilidades mentales de los alumnos, como la clasificación, la percepción de similitudes y diferencias, y la diferenciación entre la información.

3. Ir más allá del conocimiento y los datos disponibles de los alumnos.

4. Asistir en la formación de estudiantes que tengan la capacidad de innovar, inventar y producir conocimiento.

Las características de Discovery Learning:

El método de aprendizaje por descubrimiento tiene características que lo distinguen de otros métodos. Estas características como mencioné (Muhammad, 146, 2010) incluyen:

1. Hacer que el alumno sea el centro del proceso educativo en lugar del maestro creando el ambiente y las condiciones necesarias que hacen que el alumno descubra la información por sí mismo en lugar de prepararla del libro o del maestro.

2. Destaca los procesos mentales (observación, interpretación, comparación, predicción, razonamiento y organización).

3. Énfasis en el alumno y no en el material aprendido.

4. Considerar el proceso educativo como continuo sin terminar y no terminar con el estudio de un tema en particular, pero el estudio de este tema es el punto de partida para otros estudios vinculados a él.

5. Tome las características de la situación educativa integrada que coloca al alumno en la posición del descubridor, no del puerto, pone ante él los problemas que le interesan y necesitan una solución y tiene que planearse para resolver.

6. Orlikh y otros. Se agregó un criterio clave para cualquier trabajo descrito como descubrimiento de que el buscador debe transmitir "qué" y "cómo" a los demás. Si alguien descubrió una mina y no se lo contó a nadie, no descubrió nada.

Muhammad (2009, 59) mencionó otras características del aprendizaje por descubrimiento:

A. Desarrolle el talento de los alumnos y practique sus pasatiempos.

B. Le interesan las preguntas "Divergentes", que se pueden llamar preguntas amplias o abiertas en lugar de preguntas con aspectos complejos "Convergentes", que se pueden llamar preguntas específicas o preguntas pendientes.

El concepto de hablar: es necesario distinguir entre hablar y hablar; donde el habla en el idioma es el nombre de todo lo que habla y cuando la gramática es lo que encuentro en dos cosas; Una palabra es un sonido que contiene letras y palabras. Además del beneficio 
es el significado del significado en la mente del hablante, el habla y la descripción del proceso de producción del lenguaje, pero hablar es el proceso de producir el mismo lenguaje de acuerdo con los sistemas de sonido, morfología, gramática y semántico, llegamos a la conclusión de que el discurso es para abordar el discurso y realizado correctamente, y el final del sermón se convierte en palabras

\section{Metodología y procedimientos de investigación:}

Primero: Metodología de la investigación: El investigador adoptó el método experimental, ya que es el método apropiado para lograr el objetivo de su investigación.

Segundo: Diseño experimental: La elección del diseño experimental adecuado para la naturaleza de la investigación es esencial, ya que es importante para el éxito de la investigación y la verificación de hipótesis y la precisión de los resultados, ya que garantiza al investigador el camino correcto y lo toma de la mano. a los resultados correctos.

El investigador adoptó un diseño experimental con un ajuste parcial para dos grupos, ya que se ajusta a sus procedimientos de investigación. La tabla (1) muestra que:

Tabla (1) Diseño experimental de la investigación.

\begin{tabular}{llll}
\hline El grupo & Variable independiente & $\begin{array}{l}\text { Herramienta de } \\
\text { búsqueda }\end{array}$ & $\begin{array}{l}\text { La variable } \\
\text { dependiente }\end{array}$ \\
\hline Experimental & $\begin{array}{l}\text { Escultismo Orientado a la } \\
\text { Estrategia }\end{array}$ & $\begin{array}{l}\text { Prueba de } \\
\text { elegibilidad }\end{array}$ & $\begin{array}{l}\text { Habilidades del } \\
\text { habla }\end{array}$ \\
Controlar & Forma tradicional & & \\
\hline
\end{tabular}

\section{Tercero: Busca la comunidad y su muestra:}

Comunidad de búsqueda: La comunidad de investigación actual consiste en un "día" de escuela intermedia para niños, que contiene dos divisiones y más, que se encuentra en el centro de la ciudad de Babilonia para el año académico (2017-2018). Por lo tanto, el investigador visitó la Dirección General de Educación en la ciudad de Babilonia (División de Estadísticas) para averiguar el número de escuelas diurnas para niños ubicadas en el centro de esta ciudad. El número fue de aproximadamente (10) escuelas.

Muestra de investigación: La muestra del estudio es parte de la comunidad de investigación original, seleccionada de diferentes maneras, de una manera que representa a la sociedad original y logra los propósitos de la investigación. El investigador se aleja de las dificultades de estudiar la sociedad original en su conjunto. La muestra de investigación actual se divide en lo siguiente:

Muestras de escuelas A: Después de que el investigador identificó las escuelas cubiertas por la investigación, el número era de (10) escuelas, el investigador eligió la escuela de día intermedio Al-Hakim para niños de forma aleatoria *. 


\section{ORANGE JOURNAL}

Muestra de B-Students: Después de que el investigador seleccionó al azar el día de la escuela intermedia de Al-Hakim para varones, el investigador visitó la escuela después de obtener una adición de la Dirección General de Educación en la ciudad de Babilonia para facilitar su misión, encontró que contiene dos divisiones para el primer grado intermedio, a saber (A, B), el investigador eligió estas divisiones (A, B) al azar para ser la muestra de la investigación, (A) era el grupo experimental, (b) era el grupo de control, como el número de estudiantes (51), incluidos 25 estudiantes en la División A y 26 estudiantes en la División B, después de la exclusión de los (3) estudiantes reprobados, (1) estudiantes de la División A y (2) estudiantes de la División B , la muestra final fue sobre (48) estudiantes. La exclusión del investigador por su creencia de que los estudiantes que han experimentado la experiencia, la experiencia puede afectar negativamente la precisión de los resultados estadísticamente, manteniéndolos en clase para mantener el sistema escolar y la continuación de su enseñanza. La tabla (2) presenta lo siguiente:

Tabla (2) Preparar a los alumnos de los dos grupos de investigación.

\begin{tabular}{lllll}
\hline El grupo & División & $\begin{array}{l}\text { Numero de } \\
\text { estudiantes }\end{array}$ & $\begin{array}{l}\text { Número de } \\
\text { estudiantes que } \\
\text { reprobaron }\end{array}$ & $\begin{array}{l}\text { El número de estudiantes } \\
\text { después de la exclusión } \\
\text { de los desaparecidos }\end{array}$ \\
\hline $\begin{array}{l}\text { Experimental } \\
\text { Controlar }\end{array}$ & UNA & 25 & 1 & 24 \\
\hline
\end{tabular}

Cuarto: Con el fin de verificar la seguridad interna del diseño experimental, la paridad entre los dos grupos (experimental y control) se llevó a cabo en variables que se cree que interfieren con el efecto de la variable independiente en la variable dependiente, que puede Afectar los resultados del experimento:

1. La edad cronológica de los estudiantes se calcula en meses.

2. El logro educativo de los padres del alumno.

3. El logro educativo de las madres.

4. Calificaciones del examen de medio año para el idioma árabe para el año académico 2017-2018.

El investigador obtuvo datos en las variables $(1,2,3)$ de la información de la tarjeta escolar. Además, el propio investigador tomó los datos y los verificó con los propios estudiantes a través de un formulario de información que se les distribuyó en el Apéndice (4). Obtuvo los datos requeridos del registro de puntaje escolar.

\section{Herramientas de búsqueda y su construcción:}

1- Escala de habilidades del habla: El objetivo es identificar las habilidades de habla apropiadas para los estudiantes en el primer grado intermedio; Desarrollar una prueba para medirlos antes y después de aplicar la estrategia.

Escala de habilidades del habla: El investigador adoptó la escala de habilidades del habla preparada por (Rashidi '2014), que contenía diez habilidades, que se presentó a los 
árbitros para verificar su veracidad y la validez de las formulaciones del lenguaje; el esquema muestra que:

\begin{tabular}{ll}
\hline Norte & \multicolumn{1}{c}{ Habilidades } \\
\hline 1 & Las voces hablan correctamente y con claridad. \\
2 & Distinguir movimientos cortos y largos al pronunciar. \\
3 & Se hace una distinción al pronunciar sonidos similares. \\
4 & Los tonos se utilizan correctamente y apropiadamente. \\
5 & Distinguir entre pronunciación y otros fenómenos lingüísticos. \\
6 & El sistema correcto se utiliza para instalar la oración al hablar \\
7 & Expresar ideas oralmente usando las flores gramaticales y cognitivas \\
8 & apropiadas. \\
& Se inicia en la expresión oral de ideas sin dejar de evadir un déficit del \\
9 & habla. \\
& Las ideas se expresan en el vocabulario apropiado de acuerdo con las \\
10 & reglas de selección y composición. \\
\hline
\end{tabular}

2. Las habilidades para hablar con la tarjeta de notas son adecuadas para los estudiantes de la primera clase intermedia: se construyó una tarjeta para observar el desempeño de los estudiantes en las habilidades para hablar cuando los estudiantes están expuestos a preguntas orales y están destinadas a responderlas, y el observador a su vez, el Registro de calificaciones en la tarjeta según la respuesta del alumno.

Fuentes de preparación de la tarjeta: El investigador prepara una tarjeta de notas para hablar a través de:

1. Estudie los estudios anteriores relacionados con el tema de la investigación actual, y benefició al investigador en la formulación de los datos de la tarjeta y las instrucciones de uso y clasificación.

2. Habilidades de habla y habla alcanzadas en la lista de habilidades de habla final.

3. Descripción de la tarjeta: La tarjeta de notas incluía las habilidades para hablar, las habilidades totales alcanzadas en la lista de habilidades para hablar en la forma final y diez habilidades, la diversidad de calificaciones está de acuerdo con el desempeño del estudiante:

\begin{tabular}{lll}
\hline \multicolumn{2}{c}{ Evaluación } \\
\hline Muy bien & Bueno & Aceptado \\
3 & 2 & 1 \\
\hline
\end{tabular}

Validación de la tarjeta : La tarjeta presentada a un grupo de árbitros especializados en planes de estudio y métodos de enseñanza del idioma árabe, para determinar la validez de la solicitud y expresar sus opiniones sobre la idoneidad de las calificaciones para el desempeño del estudiante, tal como es válidos para los estudiantes de la primera clase 


\section{ORANGE JOURNAL}

intermedia, los expertos acordaron en su aprobación de la preparación de la aplicación y que es válido para medir lo que se preparó para su medición.

Verificación de la tarjeta: El investigador adoptó el método de acuerdo de los observadores para garantizar la estabilidad de la tarjeta, en el que dos o más observadores observan el desempeño del alumno, y el investigador observó el desempeño de cinco estudiantes en cooperación con un colega para Él para estudiantes de la escuelaintermedia de Rafidain para niños el 2/10/2018 como:

1. Cada observador se sienta en un lugar que le permite ver y escuchar al alumno con la independencia de cada observador y asignar una nota a cada alumno en particular.

2. Cada observador comienza a registrar datos simultáneamente y termina al mismo tiempo con la independencia de cada observador.

3. Descargue los datos obtenidos por el investigador y los datos obtenidos por el otro observador. Sobre la base de lo anterior, el investigador calculó el coeficiente de estabilidad a través de la aplicación de la ecuación (cobre), que establece:

\section{Cantidad de veces acordada}

Relación de acuerdo $=$ $\mathbf{x} 100$

\section{Número de veces de acuerdo + número de veces de desacuerdo}

La siguiente tabla muestra el número de veces de acuerdo y desacuerdo entre los observadores y la proporción del acuerdo: Tabla (3). La proporción de acuerdo y la diferencia entre los observadores en la observación del rendimiento de los estudiantes para hablar en árabe:

\begin{tabular}{lllll}
\hline estudiantes & $\begin{array}{l}\text { Número de } \\
\text { habilidades }\end{array}$ & $\begin{array}{l}\text { Número de } \\
\text { acuerdos }\end{array}$ & $\begin{array}{l}\text { Número de } \\
\text { desacuerdos }\end{array}$ \\
\hline primero & 24 & 17 & 7 & $70 \cdot 83 \%$ \\
Segundo & 24 & Dieciséis & 8 & $66 \cdot 66 \%$ \\
Tercero & 24 & 17 & 8 & $70 \cdot 83 \%$ \\
Cuarto & 24 & 18 & 6 & $75 \%$ \\
Quinto & 24 & Dieciséis & 8 & $66 \cdot 66 \%$ \\
\hline
\end{tabular}

La tabla anterior muestra que el coeficiente de estabilidad osciló entre $66.66 \%$ y $75 \%$, lo que se considera una buena relación.

Procedimientos para la implementación del experimento: Después de la preparación de las herramientas de búsqueda y para verificar su validez y estabilidad, se estableció el grupo de búsqueda. Donde el grupo experimental consistió en (24) estudiantes de estudiantes intermedios, escuela intermedia Hakim y (24) estudiantes del grupo de control. El investigador aplicó la prueba tribal de la tarjeta de habilidades de observación en los dos grupos de investigación el 2/10/2018 y después de completar los procedimientos de aplicación tribal a los dos grupos de investigación. El investigador 
calculó los resultados de las pruebas y los comparó para verificar la equivalencia de los dos grupos.

\section{Resultados de la investigación}

Procesamiento estadístico e interpretación de los resultados: Para responder la primera pregunta: ¿Cuáles son las habilidades de habla apropiadas para los estudiantes de escuela intermedia?

La lista de habilidades para hablar se finalizó como:

\begin{tabular}{ll}
\hline Norte & \multicolumn{1}{c}{ Habilidades } \\
\hline 1 & Las voces hablan correctamente y con claridad. \\
2 & Distinguir movimientos cortos y largos al pronunciar. \\
3 & Se hace una distinción al pronunciar sonidos similares. \\
4 & El tono y el tono se usan correctamente y apropiadamente. \\
5 & Distinguir entre pronunciación y otros fenómenos lingüísticos. \\
6 & El sistema correcto se utiliza para instalar la oración al hablar \\
7 & Expresar ideas oralmente utilizando fórmulas gramaticales y cognitivas \\
8 & $\begin{array}{l}\text { Copropiadas. } \\
\text { Comienza en una expresión oral de ideas sin interrupción que expresa un }\end{array}$ \\
9 & $\begin{array}{l}\text { Las ideas se expresan en el vocabulario apropiado de acuerdo con las reglas } \\
\text { de selección y composición. }\end{array}$ \\
10 & Coherencia entre significados e ideas y la forma lingüística apropiada \\
\hline
\end{tabular}

Para responder a la segunda pregunta: ¿Cuáles son las habilidades de habla apropiadas para los estudiantes de escuela intermedia?

La tarjeta de notas se aplicó a los grupos experimental y de control para determinar la paridad de los dos grupos. El investigador utilizó la prueba t para extraer la media aritmética y la desviación estándar de las puntuaciones totales de los estudiantes en la aplicación tribal de la nota de las habilidades de habla árabe de los dos grupos. Alcanzó (1.157) habilidades de habla; a un nivel era mayor que (0.05). Los dos grupos son homogéneos e iguales porque el promedio aritmético del grupo de control y la media experimental del grupo experimental son convergentes.

Para responder a la tercera pregunta, que es: ¿Cuál es la base de la estrategia basada en el portal de comunicación para desarrollar las habilidades de hablar entre los estudiantes de escuela intermedia? ¿Cuáles son sus componentes?

Los fundamentos de la estrategia propuesta se basan en la orientación de exploración y sus componentes que se identificaron de la siguiente manera:

- Pasos y componentes de la estrategia propuesta:

- Los pasos que prepararé una lección de la primera clase intermedia. 


\section{ORANGE JOURNAL}

El proceso de enseñanza con el descubrimiento en tres etapas es:

La primera etapa (planificación): El maestro en esta etapa debe identificar:

1. El concepto debe ser enseñado a los estudiantes con precisión...

2. Los ejemplos son relevantes para el concepto a presentar, pertenezcan o no.

Etapa 2 (presentación): El maestro en esta etapa debe hacer:

1. Presentación de ejemplos del concepto a enseñar a los alumnos...

2. Asignar estudiantes para descubrir las relaciones, asociaciones y características comunes de cada grupo...

3. Elaborar el concepto en forma precisa.

Etapa 3 (evaluando): El maestro en esta etapa:

1. Proporcionar a los estudiantes retroalimentación sobre su comprensión del concepto.

2. Pedir a los alumnos que mencionen otros ejemplos.

Para responder a la cuarta pregunta: ¿Cuál es la efectividad de la estrategia propuesta basada en el enfoque de exploración en el desarrollo de las habilidades de habla de los estudiantes de la primera clase intermedia?

Para responder la cuarta pregunta, la efectividad de la estrategia propuesta se basa en el enfoque de scouting en el desarrollo de las habilidades de habla de los estudiantes. Los resultados del balance entre los grupos experimental y de control en las habilidades de la tarjeta de notas en la post-aplicación: el investigador usó la prueba $\mathrm{T}$ para calcular la diferencia entre los puntajes promedio de los grupos experimental y de control en la aplicación de habilidades del habla, y la siguiente tabla muestra lo siguiente:

La diferencia entre los puntajes promedio de los grupos experimental y de control en la aplicación posterior de la tarjeta de notas utilizando habilidades de habla con el uso de la prueba T:

\begin{tabular}{|c|c|c|c|c|c|c|c|}
\hline \multirow[t]{2}{*}{ Habilidades } & \multicolumn{2}{|l|}{ Control (24) } & \multicolumn{2}{|c|}{ Exprimental } & \multirow[t]{2}{*}{$\overline{\mathrm{T}}$} & \multirow{2}{*}{$\begin{array}{l}\text { Nivel de } \\
\text { significancia }\end{array}$} & \multirow[t]{2}{*}{ Resultado } \\
\hline & $M^{1}$ & $\mathrm{Un}^{1}$ & M2 & A2 & & & \\
\hline $\begin{array}{l}\text { Habilidades } \\
\text { para hablar }\end{array}$ & dieciséis ، 13 & 2 '2 & $23 ، 50$ & $1 \cdot 7$ & $12 \cdot 550$ & $0 \cdot 01$ & Función \\
\hline
\end{tabular}

De la tabla anterior, hay una diferencia estadísticamente significativa entre el promedio de los grupos experimental y de control en la prueba de habilidades del habla donde el valor de $\mathrm{T}(12,550)$ estaba en un nivel inferior a $(0,01)$. La diferencia es a favor del grupo experimental, la aritmética promedio alcanzó a $(\mathrm{I} 23,50)$, mientras que la media aritmética del grupo de control alcanzó a $(16,13)$. 
Esto se explica por el papel de la estrategia propuesta basada en el enfoque de exploración en el desarrollo de las habilidades de habla árabe de los primeros estudiantes intermedios estudiados a través de esta estrategia y sus contenidos en su enfoque en las habilidades de expresión específicas dentro de cada lección.

\section{Conclusiones}

A la luz de los resultados del presente estudio, los investigadores pueden concluir lo siguiente:

1. Enseñar de acuerdo con la estrategia orientada Scout ayuda al maestro a preparar planes que son de buen conocimiento, educativos, prácticos y evaluativos.

2. El uso de la estrategia orientada a Scout hace que el alumno sea el centro del proceso educativo y el maestro es el guía y la guía para el curso de la lección.

3. El investigador observó la interacción positiva dentro de la clase para los estudiantes del grupo experimental en lugar del grupo de control, como lo confirmaron los resultados.

\section{Recomendaciones}

A la luz de los hallazgos de la investigación actual, se recomienda lo siguiente:

1. Capacitar a los maestros es sobre cómo emplear estrategias orientadas al Movimiento Scout.

2. Para obtener beneficios en la redacción de libros de texto a partir de los principios y estrategias de la orientación scouting.

\section{Sugerencias}

En vista del impacto de la estrategia orientada al Movimiento Scout en el desarrollo de habilidades de habla árabe y como complemento de la investigación actual, el investigador propone lo siguiente:

1. Llevar a cabo estudios similares para ver el impacto de la estrategia orientada al Movimiento Scout en el desarrollo de las habilidades del habla en diferentes etapas de estudio.

2. Llevar a cabo estudios similares para descubrir el efecto de la estrategia orientada al scouting en el rendimiento académico en diferentes muestras.

Información financiera: No hay información financiera.

Conflicto de intereses: Ninguno a declarar. 


\section{ORANGE JOURNAL}

\section{Liquidación ética}

Todos los protocolos experimentales se aprobaron en la Facultad de Odontología, Universidad de Babilonia, ciudad de Hillah, Irak y todos los experimentos se llevaron a cabo de acuerdo con las directrices aprobadas.

\section{Referencias}

Amoudi, Hala Saeed Ahmed Baqader (2003). La efectividad del uso del método de investigación e investigación en la enseñanza de experimentos de laboratorio en bioquímica sobre el rendimiento académico en la Facultad de Educación, Makkah Al Mukarramah (mensaje no publicado). Universidad de Umm Al Qura, Arabia Saudita. Ayashi, Jamal al-Din (1977). Estudios en Sharia, Literatura y Sociología, 1, Fanzi Press, Túnez.

Hassan, Tamam (2001). Lenguaje entre normativo y descriptivo, I 4, Dar Al Ketub, El Cairo - Egipto.

Jabir, Waleed Ahmed (2005 a). Métodos generales de enseñanza, planificación y aplicaciones educativas. Amman: Dar Al Fikr para la publicación y distribución.

Jabir, Walid Ahmed (2005 b). Métodos generales de enseñanza, planificación y aplicaciones educativas. Amman: Dar Al Fikr para la publicación y distribución.

Jamal, Muhammad Jihad, y otros (2006). Pensamiento del habla, Casa del libro de la universidad, Emiratos Árabes Unidos.

Madkour, Ali Ahmed (2008). Enseñanza de artes del lenguaje árabe, I3, Dar Al Fikr Al Arabi, El Cairo.

Madkour, Ali Ahmed (2009). Métodos de enseñanza de la lengua y la teoría árabes, 1, Dar Al-Masirah, Amman, Jordania.

Mar'i, Tawfiq Ahmed y Muhammad Mahmoud Al-Hila (2002). Métodos generales de enseñanza, I 1, Dar Al Masirah, Amman - Jordania.

Mohammed, Safaa Ahmed (2009). Descubrimiento de la educación y conceptos científicos. El Cairo: El mundo de los libros para imprimir, publicar y distribuir... 